\title{
BMJ Open Medical professionals' perspectives on prescribed and over-the-counter medicines containing codeine: a cross-sectional study
}

\author{
Michelle Foley, ${ }^{1}$ Tara Carney, ${ }^{2}$ Eileen Rich, ${ }^{2}$ Charles Parry, ${ }^{2}$ \\ Marie-Claire Van Hout, ${ }^{1}$ Paolo Deluca ${ }^{3}$
}

To cite: Foley M, Carney T, Rich $\mathrm{E}$, et al. Medical professionals' perspectives on prescribed and over-thecounter medicines containing codeine: a cross-sectional study. BMJ Open 2016;6: e011725. doi:10.1136/ bmjopen-2016-011725

- Prepublication history and additional material is available. To view please visit the journal (http://dx.doi.org/ 10.1136/bmjopen-2016011725).

Received 29 February 2016 Revised 21 June 2016 Accepted 23 June 2016

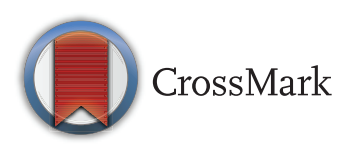

${ }^{1}$ School of Health Sciences, Waterford Institute of Technology, Waterford, Ireland

${ }^{2}$ Alcohol, Tobacco and Other Drug Research Unit, South African Medical Research Council, Cape Town, South Africa

${ }^{3}$ Institute of Psychiatry,

Psychology and

Neuroscience, King's College London, London, UK

Correspondence to Dr Michelle Foley; mfoley@wit.ie

\section{ABSTRACT}

Objectives: To explore prescribing practitioners' perspectives on prescribed codeine use, their ability to identify dependence and their options for treatment in the UK.

Design: Cross-sectional design using a questionnaire containing closed-ended and open-ended items.

Setting: A nationally representative sample of prescribing professionals working in the UK.

Participants: 300 prescribing professionals working in primary care and pain settings.

Results: Participants stated that they regularly reviewed patients prescribed codeine, understood the risks of dependence and recognised the potential for codeine to be used recreationally. Over half the participants felt patients were unaware of the adverse health consequences of high doses of combination codeine medicines. One-quarter of participants experienced patient resentment when asking about medicines containing codeine. Just under $40 \%$ of participants agreed that it was difficult to identify problematic use of codeine without being informed by the patient and did not feel confident in identification of codeine dependence. Less than $45 \%$ of all participants agreed that codeine dependence could be managed effectively in general practice. Slow or gradual withdrawal was the most popular suggested treatment in managing dependence. Education and counselling was also emphasised in managing codeine-dependent patients in primary care.

Conclusions: Communication with patients should involve assessment of patient understanding of their medication, including the risk of dependence. There is a need to develop extra supports for professionals including patient screening tools for identifying codeine dependence. The support structure for managing codeine-dependent patients in primary care requires further examination.

\section{INTRODUCTION}

Pain is a common reason for accessing primary care services. It is estimated that 14 million people in the UK suffer from long-term pain, ${ }^{1}$ with patients presenting with headaches

\section{Strengths and limitations of this study}

- This is the first study examining medical professionals' perceptions of medicines containing codeine across the UK.

- The study used a questionnaire design with closed-ended and open-ended items relating to prescribed and over-the-counter medicines containing codeine and included questions on dependence and treatment options in practice.

- Professionals involved in the prescribing of codeine were accessed across the UK using the principles of stratified random sampling.

- Response rates were lower than expected and the study was unable to access the full population of nurse prescribers.

- This study is cross-sectional and therefore does not describe how the situation might change over time.

thought to account for 1 in 25 of primary care consultations, ${ }^{2}$ and musculoskeletal pain accounting for 1 in $7 .{ }^{3}$ Opioids are widely used in pain management with codeine being the second most widely prescribed opioid medicine in general practice. ${ }^{4}$ In 2012 , it was estimated that 640 codeine prescriptions per 1000 patients were dispensed in the $\mathrm{UK}^{5}$ Additionally, medicines containing codeine can be purchased over the counter (OTC) in pharmacies, albeit with restrictions. ${ }^{6}$ Medicines containing codeine (up to $12.8 \mathrm{mg}$ per unit dose) may only be sold in the UK under the supervision of a qualified pharmacist. There are restrictions on the quantity of tablets permitted for sale in a single transaction and the product is not available for selfselection, although advertising of codeinecontaining medicines is permitted in the pharmacy and on national media. A sale may be refused if there is suspicion that the buyer is misusing codeine. Currently, the nature and extent of OTC codeine use and misuse in the UK is not widely reported. 
Treatment with opioid medication is thought to be effective in the treatment of moderate pain for acute and short periods of $<6$ months. ${ }^{7}$ Current scientific evidence measuring the efficacy of codeine over other alternative medications for chronic and longer term pain remains inconclusive. ${ }^{8}$ There are limited studies examining the efficacy of low doses of codeine found in many prescribed and OTC medicines $(<12.8 \mathrm{mg}$ per unit dose). While several Cochrane reviews have evaluated the efficacy of codeine, these are principally confined to acute postoperative pain at high doses $(60 \mathrm{mg})$. Some studies show codeine as clinically useful in some patients. A Cochrane review using 14 studies comprising 926 participants compared the use of single-dose oral paracetamol plus codeine with the same dose of paracetamol alone for postoperative pain in adults. The review concluded that the addition of codeine provided effective pain relief to $\sim 10 \%$ more participants than the same dose of paracetamol alone. The use of codeine in combination with paracetamol was found to extend the duration of analgesia by $\sim 1$ hour. ${ }^{9}$ A recent Cochrane review found that the combination of ibuprofen $400 \mathrm{mg}$ plus codeine 25.6-60 mg demonstrated effective analgesic efficacy in postoperative pain; however, very limited data suggest that the combination is better than the same dose of either drug alone. ${ }^{10}$ Equally, the use of codeine-containing syrups in supressing cough appears to lack positive scientific basis and is not widely discussed in the current literature.

While codeine is considered a weak opiate, it carries an identified abuse potential. Development of tolerance on regular or excessive use appears within a short time frame. ${ }^{11}$ Literature reports increasing trends in the misuse of codeine, including OTC preparations, which appears to incur significant negative epidemiological, social and economic consequences. ${ }^{12-16}$ Related harms in terms of morbidity and mortality are documented, ${ }^{12}$ along with monetary costs associated with indirect effects on healthcare, prevention and treatment. ${ }^{17}$

Treatment of codeine dependence is varied and does not appear to be well documented in the literature. Guidance on options for opioid dependence is evident but appears non-specific to weak opioids such as codeine. Summary statistics for codeine dependence obtained from treatment providers in the UK suggest that codeine as the primary and secondary substance of misuse is extremely low at 2.2\% (4248 individuals) of all those entering addiction treatment services in the period 2013-2014. ${ }^{18}$ These statistics may give an impression that codeine dependence is not an issue warranting attention compared with other substances of misuse; however, treatment for codeine dependence conducted in primary care does not appear in national treatment surveillance systems. What is of particular concern is that codeine-dependent patients appear to function well within the range of perceived normality, carrying out the functions of normal daily living. ${ }^{19}$ Evaluating as to whether medical professionals are equipped to detect and manage patients presenting with codeine dependence is therefore an area of considerable importance.

Studies have called for further research on the experiences and concerns of medical professionals around use of medicines containing codeine, with particular focus on their experiences, challenges, perspectives and practices. ${ }^{20}$ The aim of the study was to garner information regarding prescribing practitioners' perspectives on prescribed and OTC codeine use, their ability to identify dependence and options for treatment in the UK. This study was part of a larger study examining OTC and prescribed misuse of codeine medicines in the Republic of Ireland, UK and South Africa and was funded by the European Commission (http://www.codemisused.org).

\section{METHOD}

\section{Recruitment of participants and study procedures}

The study involved a cross-sectional design and took place between May 2014 and April 2015 using an online questionnaire (see figure 1). A nationally representative sample of medical practitioners in the UK was facilitated through Specialist Info, a medical directory specialist. A random list of 1000 practice managers was generated by strata using proportionate numbers of general practitioners (GPs) present in each country (Wales $n=48$, Northern Ireland $n=36$, Scotland $n=98$, England $n=818$ ). Each practice manager was asked to recruit at least one GP from their respective practice to participate in the study. Each practice manager was sent a reminder $\sim 2-$ 4 weeks later. A total of 150 pain specialist physicians' email records were also retrieved from Specialist Info and these were subsequently sent the link to the survey. In order to have representation from nurse prescribers, a previous distribution list of 98 pain specialist nurses was used. ${ }^{21}$ These participants were asked to indicate their agreement to be added to the email distribution list. In total, 54 agreed to participate and the link to the survey was then provided. In total, this process resulted in 136 participants completing the online questionnaire. A further 65 GPs were recruited through GP target sessions. A second random sample of 1000 practice managers was generated, checked for duplicates and emailed as previously described. A follow-up reminder was distributed 46 weeks later. This resulted in the completion of a further 98 questionnaires. The indicated time to complete the questionnaire was $\sim 10 \mathrm{~min}$, and this was specified in the email correspondence and prior to proceeding with the questionnaire. Information on the study was provided and informed consent was obtained prior to proceeding online. Participants were advised to complete the questionnaire only once. Dissemination activities, including circulars and newsletters, were used to encourage participation in the study. Ethical approval was granted by King's College London Ethics Committee (PNM/13/14-75). 


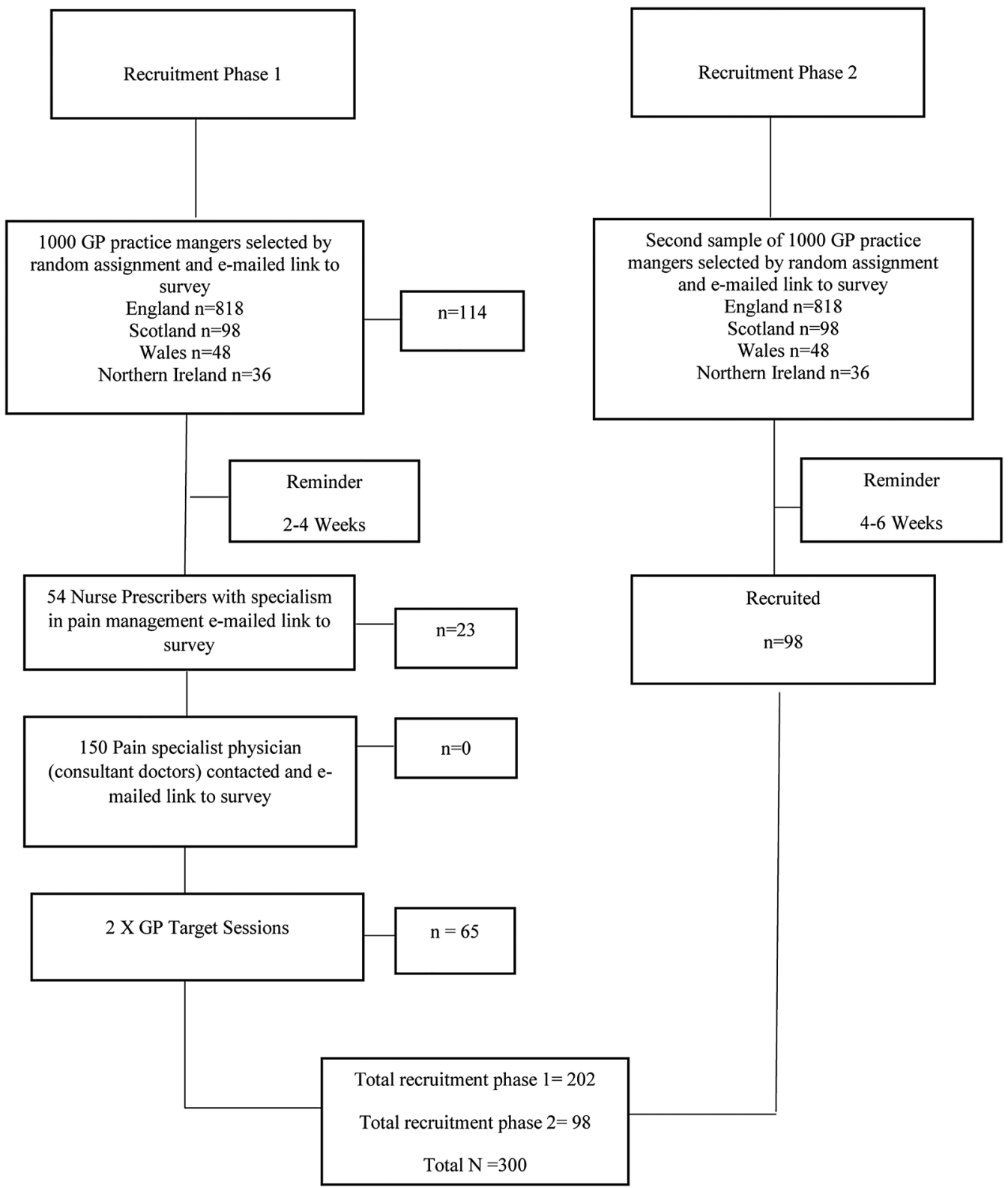

Figure 1 Recruitment of participants to the study. GP, general practitioner.

\section{Data collection methods}

We collected the data using an online questionnaire using the survey tool http://www.onlinesurveys.ac.uk. Questions were developed to bridge gaps in knowledge following a comprehensive search of the literature. ${ }^{4}$ Eight questions were added to collect demographic information in order to establish the representativeness of the respondents. The questionnaire comprised a combination of closed-ended and open-ended items, on prescribed and OTC medicines containing codeine, and included questions on triggers for suspecting codeine misuse, managing codeine-dependent patients and reasons for referral. Participants were provided with an opportunity to add additional comments at the end of the questionnaire and knowledge of innovations on preventing medicine misuse (see online supplementary information for full details of questionnaire). A paper edition of the questionnaire was made available at GP target sessions in two separate locations (continuing professional development). GPs had the option to complete the questionnaire online via the survey link or complete the paper copy and return to the researcher at the end of the target session. GPs were also informed that they could return by post and were provide with a return envelope on request.

\section{Data analysis}

Data were downloaded directly from the online data capture page to SPSS V.21. Data captured in paper format were entered manually and combined with the data captured online. Data were screened and checked for errors. Data were then examined descriptively using 
Table 1 Demographic details and information pertaining to profession, location and specialist training

\begin{tabular}{|c|c|c|}
\hline & \multicolumn{2}{|l|}{$\mathrm{N}=300$} \\
\hline & $\begin{array}{l}\text { Frequency } \\
\text { (f) }\end{array}$ & $\begin{array}{l}\text { Per } \\
\text { cent }\end{array}$ \\
\hline \multicolumn{3}{|l|}{ Gender } \\
\hline Male & 140 & 46.7 \\
\hline Female & 160 & 53.3 \\
\hline Age & \multicolumn{2}{|c|}{$\begin{array}{l}\text { Median } 48 \text { years, } \\
\text { range } 25-68 \text { years }\end{array}$} \\
\hline \multicolumn{3}{|l|}{ Profession } \\
\hline General practitioner & 238 & 79.3 \\
\hline $\begin{array}{l}\text { Independent/supplementary/ } \\
\text { nurse prescriber }\end{array}$ & 23 & 7.7 \\
\hline Specialist in family medicine & 31 & 10.3 \\
\hline Specialist in pain management & 0 & 0 \\
\hline Other & 8 & 2.7 \\
\hline $\begin{array}{l}\text { Number of years as a qualified } \\
\text { practitioner }\end{array}$ & \multicolumn{2}{|c|}{$\begin{array}{l}\text { Median } 20 \text { years, } \\
\text { range } 1-48 \text { years }\end{array}$} \\
\hline $\begin{array}{l}\text { Number of consultations in an } \\
\text { average week }\end{array}$ & \multicolumn{2}{|c|}{$\begin{array}{l}\text { Median 100, range } \\
7-500\end{array}$} \\
\hline \multicolumn{3}{|l|}{ Country } \\
\hline England & 253 & 84.6 \\
\hline Wales & 15 & 5.0 \\
\hline Scotland & 23 & 7.7 \\
\hline Northern Ireland & 9 & 2.7 \\
\hline \multicolumn{3}{|l|}{ Location } \\
\hline Urban & 166 & 55.3 \\
\hline Rural & 40 & 13.3 \\
\hline Mix of both & 94 & 31.3 \\
\hline \multicolumn{3}{|c|}{ Specialist training in substance misuse } \\
\hline Yes & 89 & 29.7 \\
\hline No & 208 & 69.3 \\
\hline Did not indicate & 3 & 1.0 \\
\hline \multicolumn{3}{|l|}{ Type of substance misuse training } \\
\hline Certificate in substance misuse & 42 & 47.2 \\
\hline $\begin{array}{l}\text { Postgraduate qualification in } \\
\text { substance misuse }\end{array}$ & 13 & 14.6 \\
\hline $\begin{array}{l}\text { Continuing professional } \\
\text { development (CPD) }\end{array}$ & 12 & 13.4 \\
\hline Other & 5 & 6.3 \\
\hline Did not indicate & 17 & 19.1 \\
\hline
\end{tabular}

frequencies and percentage. The data were summarised to create two categories by combining levels of agreement and disagreement for reporting purposes. The occurrence of codeine dependence was estimated using the approximate numbers of patients suspected of having codeine dependence as indicated in the questionnaire divided by the number of consultations indicated by professionals in an average month. Referrals were estimated using the numbers indicated by participants in the questionnaire, and this figure was presented as an overall percentage of the total number of participants' referrals to secondary care for codeine dependence.

The open-ended questions were downloaded into a separate Excel sheet for content analysis. These data were examined and individual categories were created for each of the open-ended questions based on common features and dominant subjects identified in the text. The categories were discussed by two senior members of the research team and agreed. Three researchers independently coded the data (two academic researchers and one pharmacist). This was achieved by labelling each field where the content matched the associated thematic category. Intercoder reliability of the data was conducted by dialogue between three members of the research team. Each item was checked for agreement and non-agreement with the thematic categories. Where discrepancies were identified or disagreement with the category placing occurred, the researchers discussed the illustrated content. This was then resolved when two or more researchers were in agreement. Data were presented as an overall percentage of those who responded to the open-ended questions.

\section{RESULTS}

Three hundred medical professionals involved in prescribing codeine were recruited to the study, giving an overall response rate of $\sim 12.5 \%$. Table 1 details the demographic information. The mean age of participants was 47 years (range 25-68 years). The average number of years of practice was 19.52 years (range 1-48 years).

\section{Prescribed codeine}

Figure 2 illustrates statement items examining medical professionals' experiences of prescribing codeine. Percentage agreement and disagreement were used to describe the results. In total, $50 \%$ of participants showed some level of agreement that the requests for prescribed codeine were increasing. In relation to prescribing practices, $54 \%$ of participants implied that they avoided the prescribing of codeine with other depressant drugs. In total, $82 \%$ of participants agreed to some extent that they prescribed codeine following unsuccessful treatment with non-opioid analgesics. Only $12.6 \%$ of participants agreed to prescribe codeine cough linctus following unsuccessful treatment of cough with non-codeine-based medicine. Less than $20 \%$ of participants agreed that low doses of codeine, $<30 \mathrm{mg}$, are not very effective in treating mild-to-moderate pain. In total, $80 \%$ of professionals agreed to routinely reviewing patients who are prescribed codeine. In total, $27 \%$ of those responding believed that patients resented them for asking about their use of medicines containing codeine. However, most professionals disagreed with the statement in respect to 'feeling awkward' around questioning patients about their codeine use $(76.6 \%)$. Furthermore, over half showed a level of disagreement $(53.7 \%)$ with the statement "patients are aware of the adverse health consequences of high dose of combination codeine medicines'. Participants recognised the potential for codeine to be used recreationally $(81.7 \%)$. 


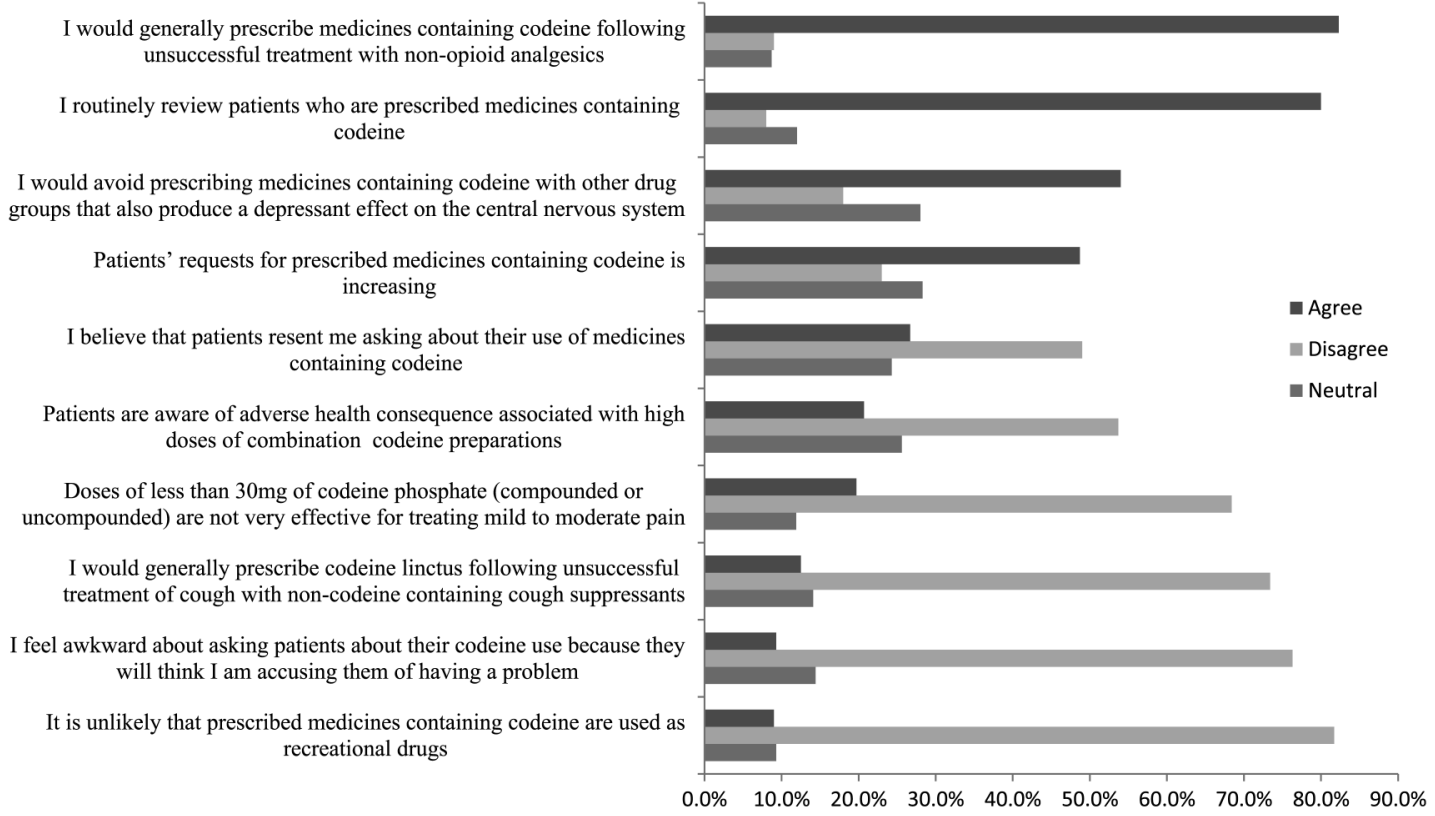

Figure 2 The level of agreement (agree strongly, agree) and disagreement (disagree strongly, disagree) and neutral responses with statements related to prescribed codeine medicine.

\section{OTC codeine}

Figure 3 shows statement items examining medical professionals' experiences of OTC codeine. Percentage agreement and disagreement were used to describe the results. A total of $76 \%$ of those responding to the questionnaire were found to routinely ask about patients' use of OTC codeine medicine, and $71 \%$ indicated that they documented the use of OTC medicines in the patients' medical notes. Concern about availability of OTC codeine in pharmacies was recorded at $45.8 \%$. Concern about the availability of OTC codeine on the internet was slightly higher at $64 \%$. However, the vast majority of practitioners agreed to some extent that the potential to buy codeine from multiple sources added significantly to the potential for misuse $(86.9 \%)$. A total of $35 \%$ showed some level of agreement that medicines containing codeine should be regulated to a prescription-only medicine, while a similar percentage $(36 \%)$ did not or had no opinion $(28 \%)$. A total of $16 \%$ of participants felt that patients were given sufficient information and there was agreement that patients were not fully aware of the risks of dependence with consumption of OTC codeine medicines $(83.8 \%)$ and believed them to be safe $(86.3 \%)$. Only $23 \%$ of practitioners agreed (or strongly agreed) that codeine was more effective than non-opioid analgesics. The potential to extract codeine from compounded formulation showed mixed levels of agreement.

\section{Dependence, screening and treatment}

Figure 4 shows responses to the various statement items examining codeine dependence. Only $8 \%$ of participants agreed that patients were not at risk of codeine dependence when they took their codeine medicine as prescribed. The majority agreed to some extent that patients did not fully understand the risk of dependence when taking prescribed medicine containing codeine $(82 \%)$. Over $40 \%$ agreed the difficulty in identification of problematic use of codeine without being informed by the patient. This corresponded with relatively high percentages of those who did not feel confident in identification of codeine dependence $(41 \%)$. When asked if women were at higher risk of development of codeine dependence, only $20.8 \%$ agreed, while $16 \%$ showed a level of disagreement. A total of $45 \%$ of all participants agreed that codeine dependence could be managed effectively in general practice.

A total $21 \%$ of participants agreed to have suitable screening methods to identify if codeine was being used inappropriately. A total of $27 \%$ of participants agreed that adequate services were in place to manage codeinedependent patients, while only $28 \%$ agreed to be fully aware of best practice in managing codeine misuse and dependence. A high proportion of participants $(70.3 \%)$ would like more instruction on the prescribing of potentially addictive medicines.

\section{Managing codeine dependence}

A total of $86 \%$ of the total participants had suspected cases of codeine dependence in practice. The median number of patients suspected of being codeine dependent was calculated as being three patients per 100 consultations. When asked if they had referred a patient to 


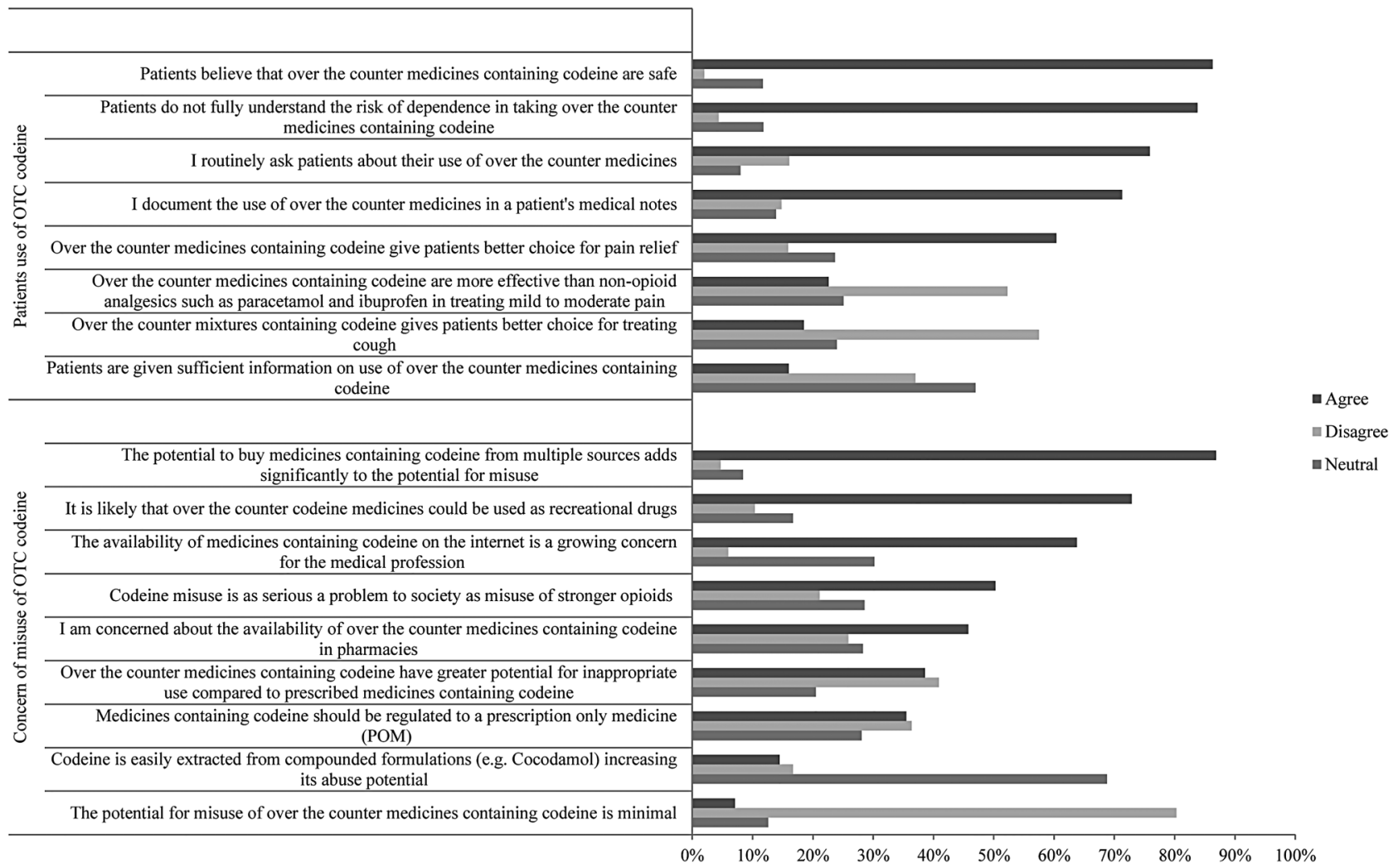

Figure 3 Percentages of agreement (strongly agree, agree) and disagreement (strongly disagree, disagree) and neutral responses with each of the statements related to over-the-counter codeine.

Patients do not fully understand the risk of dependence when taking prescribed medicines containing codeine

I would like more instruction on prescribing potentially addictive medications

Codeine dependence can be managed effectively in general practice

I find it difficult to identify problematic use of medicines containing codeine (including OTCs) without the patient first telling me

Support services are readily available in my area to help those with a codeine dependence problem

I am fully aware of best practice in managing codeine misuse and dependence

I am confident that I can identify codeine dependence in my patient

I have suitable screening methods that I use to identify inappropriate use of medicines containing codeine

Females are at higher risk of developing a codeine dependence than their male counterparts

Patients who take their codeine medication as prescribed are not at risk of developing a codeine dependence

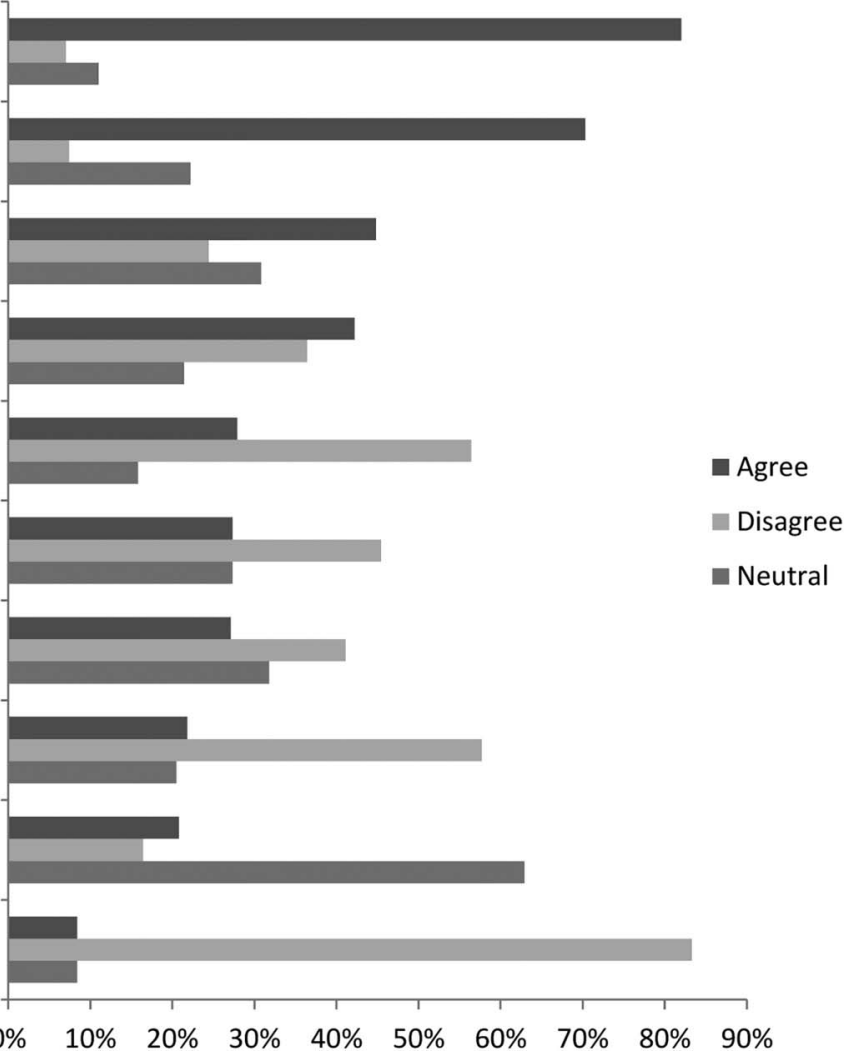

Figure 4 The level of agreement (strongly agree, agree) and disagreement (strongly disagree, disagree) and neutral responses with statement items related to codeine dependence and treatment. OTC, over the counter. 
Table 2 Main thematic categories and frequency of responses

\begin{tabular}{lr}
$\mathrm{N}=300$ & $\begin{array}{r}\text { Frequen } \\
\text { respons }\end{array}$ \\
\hline Can you describe the patient behaviours & that trigger \\
suspicion of codeine misuse? & \\
Requesting prescriptions for codeine & $212(71)$ \\
Aberrant behaviour & $89(30)$ \\
Unresolved pain/cough & $78(19)$ \\
Lost prescriptions or medications & $52(17)$ \\
Physical or psychological symptoms & $41(14)$ \\
History of addiction & $38(13)$ \\
Doctor/pharmacy shopping for codeine & $28(9)$ \\
medicines & \\
History of comorbidity & $14(5)$ \\
Socioeconomic status & $14(5)$ \\
Sex & $3(1)$
\end{tabular}

Can you describe the types of treatments that you use for patients with codeine dependence?

Slow or gradual withdrawal $153(51)$

Education/counselling 87 (29)

Referral to secondary or specialist $\quad 87$ (29)

care

Restricted prescriptions $\quad 60$ (20)

Substitution with another drug (drug 30 (10)

not specified)

Substitution using methadone/ 27 (9)

buprenorphine/other opiate drug

Substitution with non-opioid

medication

Substitution using benzodiazepines/

other antipsychotics/other

Cold turkey

What were the main reasons for referring patients to specialist treatment? ?i $^{\mathrm{ii}}$

Failure to manage patient effectively in $150(50)$

practice

Complex case requiring specialist care $186(62)$

Patient request for referral

$63(21)$

Lack of resources

specialist care for codeine dependence in the past month, $27 \%$ of participants indicated referrals to secondary/specialist care. Where referrals were indicated, the median number was one patient in a monthly period.

\section{Patient behaviours, treatment options and referral reasons}

Practitioners were asked to describe the typical patient behaviours triggering their suspicion of codeine misuse. Table 2 shows thematic categories of results displayed as a frequency and as a percentage of the total responses. Patient behaviours that triggered suspicion of codeine misuse and dependency included requesting codeine specifically by name, early requests and refills and calling the surgery at inappropriate times to request codeine medicines. The potential to obtaining codeine from multiple sources was expressed. Additional

comments reflected the difficulties in managing patients' codeine use.

It is very difficult to control patients' codeine use as they may use multiple pharmacies, buy from friends or online. I don't think we have fully woken up to the scale of the problem of opiate dependence. Especially when opiates are prescribed by pain clinics for chronic, non-cancer pain. (GP participant)

Have advised colleagues many times that this is a timebomb (not wishing to be melodramatic!) If evidence is promoted or the Daily Mail decides to champion a codeine scare we will be on the back-foot managing the fall-out and patient distress. (GP participant)

Lost prescriptions or medicines were also perceived to be an indicator of dependence, and practitioners used anecdotes to describe patient situations such as 'the dog ate my script', 'I lost my medication on the bus' or 'on holiday'. Unresolved pain was indicated as a further trigger in suspicion of dependence, and situations of hypersensitivity to pain were described in terms of headache, inadequate pain relief and indications of chronic pain not being helped by the current medication regime. Aberrant behaviours were described and included aggression, demanding codeine, reluctance to change medication and becoming very keen to obtain a script. Physical signs of misuse included restricted pupils, anxiety, constipation, gastric disturbances and irritability. Other indicators included history of comorbidity and history of addiction. Some professionals also mentioned social and economic factors including taking excessive sick leave and unemployment.

Various treatments for codeine dependence were described by practitioners (see table 2). A large proportion of respondents mentioned slow or gradual withdrawal as the suggested treatment in managing dependence. Education and counselling was also emphasised and was more often suggested with gradual withdrawal and restricted prescriptions. Additional comments were also made about the area of practitioner training in managing misuse and the requirement to improve patient knowledge on codeine consumption.

It would be helpful to have some teaching on misuse of OTC products and management there of. (GP participant)

I am very concerned about the number of people I see who take products containing a combination of codeine and paracetamol at levels that put them at high risk of liver damage as they often do not realise that taking large amounts for the psychoactive effects of the codeine means that they are likely to be unintentionally overdosing on the paracetamol. (GP participant)

Restricted prescriptions were described in terms of giving limited amounts of codeine in doses of up to 3 days or a 1-week supply. One GP spoke of the role of 
advertising and efforts to control repeat prescribing, while another also spoke of the problems of monitoring of repeat prescriptions.

We worry about codeine abuse, etc., yet Solpadeine is very heavily advertised, but it does serve a purpose in selfmedication for moderate pain. In my experience patients are started too early on high strength codeine painkillers and simply parked there via repeat prescriptions. Little effort seems to be given to follow up and monitoring usage before the patient becomes addicted to the codeine as opposed to suffering pain. (GP participant)

Big problem. We struggle to monitor repeat prescriptions closely enough. Hospitals hand out codeine and tramadol like smarties. Difficult to know how best to tackle OTC access as primary care does not have the capacity to pick up seeing all those people who self-treat appropriately with OTC meds. (GP participant)

Other suggested treatments include substitution using non-opioid analgesics, substitution with other drugs including amitriptyline and benzodiazepines, other opiates, methadone and buprenorphine. Some respondents indicated that the main treatment they used was 'cold turkey'. Referral to specialist care was also indicated, including drug and alcohol services, rehabilitation centres, psychologists, psychiatrists and pain specialists.

The primary reasons for referrals are reported in table 2 and were indicated as the inability to manage the patient effectively in primary care or that the patient was a complex case requiring specialist care. Complex cases were described as those with multiple issues, including other drugs and alcohol misuse, pregnant patients and other comorbidities, usually related to mental health. Some sentiment was also expressed about other drugs of misuse.

Whilst codeine is misused, I am more concerned with heroin and cocaine misuse. (GP participant)

We also have big problems with dihydrocodeine, oxycodone, tramadol, pregabalin and gabapentin. (GP participant)

Patient's request to be referred for specialist treatment was also a common response. Indications were also that referrals to specialist care were as a result of the lack of resources, or time during the consultation and lack of knowledge with regard to the best practice.

Some additional comments were made related to the lack of resources available to manage the problem of codeine dependence.

There is a gap between practicing perfect medicine where patients' drug use is explored in depth and the practicalities of managing time and workload. I feel that to a certain extent, exploring drug misuse in patients who are pre-contemplative is not necessarily the most effective use of time (although of course would be done in an ideal world). (GP participant)

\section{DISCUSSION}

The current study exhibited high levels of agreement that patients prescribed codeine were routinely reviewed by their medical professional and included questioning around OTC codeine use. Half of all those who responded felt that the request for codeine medicines was increasing and is substantial in raising some concern. The increasing requests for codeine may be directly as a result of newer restrictions imposed on OTC supply, forcing patients to obtain a steady source on prescription. ${ }^{21}$ Other factors contributing to the perceived increase in codeine requests may relate to changes in scheduling of other prescription opioids such as tramadol. ${ }^{22}$ In the UK, tramadol must only be supplied in quantities that do not exceed 30 days and must not be dispended on 'batch' repeat prescriptions, making refills more difficult to obtain. Codeine is currently not bound by these restrictions. While there is no specific evidence to verify that codeine consumption is increasing, a level of caution should be exercised by the prescriber when a medicine is requested specifically by name. Content analysis of the open-ended questions showed that one of the most common triggers for suspecting misuse was when codeine was requested specifically by its name or brand name.

Views regarding the effectiveness of $30 \mathrm{mg}$ of codeine showed some notable variation. The efficacy of low doses of codeine is not well documented in the literature and a greater evidence base is required to further develop indications for codeine use. ${ }^{23}$ Prescribing of codeine was indicated following unsuccessful treatment with non-opioid analgesics. However, the complexity of pain management has drawn significant attention over recent years with limited options for prescribers to avoid unpleasant side effects. ${ }^{24}{ }^{25}$ Newer drugs acting at the $\gamma$-aminobutyric acid (GABA) receptors have gathered some momentum in the treatment of chronic neuropathic pain; however, they also carry significant side effects and serious risk of misuse. ${ }^{26}$ The difficulty for any prescriber is to balance the benefits and risks of opioid use and it is feasible that codeine is considered to have lower abuse liability due to it weak opioid status. ${ }^{27}$

There is concern regarding patients' use of OTC codeine-containing medicine, and a large number of respondents in the current study believe that patients were unaware of the health consequences associated with high doses of combination codeine preparations. Equally, the respondents were of the view that patients do not understand the risk of dependence when taking prescribed medicines containing codeine. This concurs with evidence reported in the literature. ${ }^{28} 29$ Further research should identify the level and type of information patients require to make informed decisions surrounding their medicine use, both prescribed and OTC. Rescheduling of codeine has drawn significant debate and some suggestion was made in the current study to remove sales of OTC codeine altogether. ${ }^{24}$ However, without actual prevalence rates of misuse and harm, it is 
difficult to draw definitive conclusions. Equally, other prescription opioids, including codeine, are also misused, so removal of OTC codeine is unlikely to eliminate the problem entirely. Additionally, several countries across the European Union (EU) do not permit the sale of OTC codeine, ${ }^{25}$ and there is little evidence that restricting this provision has any impact on harms associated with its use. The rescheduling to a prescription-only medicine is likely to place additional pressure on GPs for self-limiting conditions and shift diversionary patterns. This may explain differences observed in the current study regarding rescheduling of codeine to a prescription-only medicine.

Risk of development of a codeine dependence in spite of taking codeine as prescribed was identified by the majority of professionals in the UK and shows that prescribers are aware of potential risks associated with its use. Detection of codeine dependence in patients appears to be problematic, with low levels of confidence expressed in detection of codeine dependence, highlighting the need for specific screening tools. ${ }^{30}$ Equally, adequate screening tools for identification of patient at risk appear lacking in practice, and practitioners indicated the desire for further training activities on prescribing potentially addictive medicines. Education of professionals on the abuse potential of codeine and the ability for individuals to extract paracetamol from compounded formulations is also warranted to enhance existing knowledge.

Currently, there are no specific clinical guidelines on managing codeine detoxification, although clinical guidance does exist under the broad umbrella of opioid detoxification. ${ }^{31}$ In the current study, an overwhelming majority of respondents indicated that they used the process of gradual withdrawal including tapering of the dose, restricting and reducing patients' prescriptions coupled with education and counselling techniques as strategies to address misuse of and dependence on codeine. A minority of participants mentioned that they would prescribe benzodiazepines and other medicines to manage codeine-dependent patients. The basis of this decision should be investigated further in light that some of the drugs mentioned come with a high abuse potential. The methods offered by respondents may well be the foundation on which to build some guidance for prescribers and pharmacists in managing detoxification, however, taking into consideration that each patient will have their own individual needs. Owing to the fact that several of the prescribers mentioned the toxicity associated with paracetamol and ibuprofen, initial treatment to reduce this risk should be the first consideration. ${ }^{11}$ The education and counselling offered and expressed by professionals in the open-ended questions could be further evaluated for its content, applicability and effectiveness in the treatment process.

Levels of referral to secondary care appear low and reflect the levels of patients entering addiction treatment indicated by the National Drug Treatment Monitoring
Service, ${ }^{18}$ and may indicate that a high proportion of patients are being effectively managed in primary care, or more seriously, not being detected at all. If this is the case, then actual prevalence rates of misuse are underreported in the national figures. The reporting of codeine misuse by general practice outside of secondary care may actually help in the estimation and prevalence of codeine and other substances of misuse in populations who remain outside of addiction services. Professionals in the current study identified a lack of support services for those identified with problems related to their codeine use. The acknowledgement of poor support requires further investigation in the context of the development of adequate services for patients within primary care, community and inpatient settings.

\section{LIMITATIONS}

There are several limitations with respect to this study. The recruitment of participants to this online survey was challenging and therefore resulted in a lower than expected response rate. The targeting of GP practice managers rather than GPs directly may have impacted on the response rate. However, due to the level of email correspondence GPs receive, filtering it in this manner may have been beneficial. There may be some criticism of the methods used to boost response; however, as a professional group, it is unlikely that participants answered the questionnaire more than once. The study is cross-sectional in nature and therefore cannot describe how the situation might change over time. It is also possible that those experiencing problem with codeine dependence in their practice may have been more likely to participate in the study. It is possible that the term misuse and dependence may have different meanings to specific responders, and while specific definitions were provided, these were contained within an additional information drop-down menu. However, one of the major strengths of the study is the fact that it responds to the increasing demand for greater information regarding codeine misuse and dependence found in the UK and provides information that can be used in future studies.

\section{IMPLICATIONS FOR PRACTICE}

Greater sources of information are required to develop policy on codeine use, misuse and treatment options in the interest of public health. This study has shed light on medical professionals' experiences in the UK and has highlighted areas of concern regarding medicines containing codeine and the need to develop specific patient screening tools. It is important to note that medicines containing codeine have utility when used appropriately; however, greater research is required to examine its indications for use in longer term pain management. Further education and training is required in the area of prescribing addictive medicines at all levels of practice. Similarly, specialist training on recognition 
of the signs and symptoms drawn from the responses of professionals in this study could help with early detection of codeine dependence. The reporting of cases of codeine dependency outside of addiction services into national registers should be considered. It is important to identify as accurately as possible the prevalence of codeine misuse and dependence in the population so that services can be adequately provided and needs addressed appropriately.

Acknowledgements The authors would like to acknowledge the CODEMISUSED project secondees who assisted with this study during their secondment periods.

Contributors All the authors contributed substantially to the study conception and design. MF, ER and CP conducted the analysis and interpretation of data. MF and PD recruited the participants. MF drafted the work and TC, M-CVH and PD revised it critically for important intellectual content. All authors gave final approval of the version to be published and agreed to be accountable for all aspects of the work in ensuring that questions related to the accuracy or integrity of any part of the work are appropriately investigated and resolved.

Funding The research leading to these results has received funding from the European Community's Seventh Framework Programme FP7/2007-2013 under grant agreement no 611736.

Competing interests None declared

Ethics approval Ethical approval by King's College London Research Ethics Committee prior to recruitment of participants (8 April 2014).

Provenance and peer review Not commissioned; externally peer reviewed.

Data sharing statement No additional data are available.

Open Access This is an Open Access article distributed in accordance with the Creative Commons Attribution Non Commercial (CC BY-NC 4.0) license, which permits others to distribute, remix, adapt, build upon this work noncommercially, and license their derivative works on different terms, provided the original work is properly cited and the use is non-commercial. See: http:// creativecommons.org/licenses/by-nc/4.0/

\section{REFERENCES}

1. Price C, Lee J, Taylor AM, et al. Initial assessment and management of pain: a pathway for care developed by the British Pain Society. Br J Anaesth 2014;112:816-23.

2. Carville S, Padhi S, Reason T, et al. Diagnosis and management of headaches in young people and adults: summary of NICE guidance. BMJ 2012;345:e5765.

3. Jordan $\mathrm{K}$, Jinks $\mathrm{C}$, Croft $\mathrm{P}$. Health care utilization: measurement using primary care records and patient recall both showed bias. $J$ Clin Epidemiol 2006:59:791-7.

4. Van Hout M, Bergin M, Foley M, et al. A scoping review of codeine use, misuse and dependence, final report. Brussels, EU: European Commission 7th Framework Programme, 2014.

5. Foley M, Rich E, Norman I, et al. Supplement to the CODEMISUSED scoping review. Waterford Institute of Technology.

6. Foley M, Harris R, Rich E, et al. The availability of over-the-counter codeine medicines across the European Union. Public Health 2015;129:1465-70.

7. Trescot $\mathrm{A}, \mathrm{Helm} \mathrm{S}$, Hansen $\mathrm{H}$, et al. Opioids in the management of chronic non-cancer pain: an update of American Society of the Interventional Pain Physicians? (ASIPP) Guidelines. Pain Physician 2008;11:S5-S62.
8. Moore R, Wiffen P, Derry S, et al. Non-prescription (OTC) oral analgesics for acute pain-an overview of Cochrane reviews. Cochrane Database Syst Rev 2015;(11):CD010794.

9. Toms L, Derry S, Moore RA, et al. Single dose oral paracetamol (acetaminophen) with codeine for postoperative pain in adults. Cochrane Database Syst Rev 2009;(1):CD001547.

10. Derry S, Karlin SM, Moore RA. Single dose oral ibuprofen plus codeine for acute postoperative pain in adults. Cochrane Database Syst Rev 2013;(3):CD010107.

11. Nielsen S, Cameron J, Pahoki S. Over the counter codeine dependence final report 2010. Victoria: Turning Point, 2010.

12. Fischer B, lalomiteanu A, Boak A, et al. Prevalence and key covariates of non-medical prescription opioid use among the genera secondary student and adult populations in Ontario, Canada. Drug Alcohol Rev 2013;32:276-87.

13. Compton WM, Volkow ND. Major increases in opioid analgesic abuse in the United States: concerns and strategies. Drug Alcohol Depend 2006;81:103-7.

14. Compton $P$, Charuvastra VC, Ling W. Pain intolerance in opioid-maintained former opiate addicts: effect of long-acting maintenance agent. Drug Alcohol Depend 2001;63:139-46.

15. Fry C, Smith B, Bruno R, et al. Benzodiazepine and pharmaceutical opioid misuse and their relationship to crime. Hobart: National Drug Law Enforcement Research Fund, 2007.

16. Benyamin R, Trescot A, Datta S, et al. Opioid complications and side effects. Pain Physician 2008;11:S105-S20.

17. Gilson AM, Ryan KM, Joranson DE, et al. A reassessment of trends in the medical use and abuse of opioid analgesics and implications for diversion control: 1997-2002. J Pain Symptom Manage 2004;28:176-88.

18. Parry $\mathrm{CDH}$, Deluca $\mathrm{P}$, Cooper R, et al. Do we have sufficient information to optimally inform regulatory or other policy decisions about medications containing codeine? Addiction 2015;110:1690-1.

19. Cooper RJ. 'I can't be an addict. I am.' Over-the-counter medicine abuse: a qualitative study. BMJ Open 2013;3:pii: e002913.

20. Sheridan J, Butler R. Prescription drug misuse: issues for primary care-final report of findings. New Zealand: The School of Pharmacy, The University of Auckland, 2008.

21. Nielsen S, Cameron J, Pahoki S. Opportunities and challenges: over-the-counter codeine supply from the codeine consumer's perspective. Int J Pharm Pract 2013;21:161-8.

22. Home Office. Scheduling of tramadol and exemptions for temazepam prescriptions. London, 2014 (cited 12 April 2016). https://www.gov.uk/government/consultations/scheduling-oftramadol-and-exemptions-for-temazepam-prescriptions

23. Furlan AD, Sandoval JA, Mailis-Gagnon A, et al. Opioids for chronic noncancer pain: a meta-analysis of effectiveness and side effects. CMAJ 2006;174:1589-94.

24. Kozma CM, Provenzano DA, Slaton TL, et al. Complexity of pain management among patients with nociceptive or neuropathic neck, back, or osteoarthritis diagnoses. J Manag Care Pharm 2014;20:455-66.

25. Macintyre PE, Schug SA. Acute pain management: a practical guide. Boca Ranton: CRC Press, 2014.

26. Huffman C, Stacey BR, Tuchman M, et al. Efficacy and safety of pregabalin in the treatment of patients with painful diabetic peripheral neuropathy and pain on walking. Clin J Pain 2015;31:946-58.

27. van Amsterdam J, Phillips L, Henderson G, et al. Ranking the harm of non-medically used prescription opioids in the UK. Regul Toxicol Pharmacol 2015;73:999-1004.

28. Frei M, Nielsen S, Dobbin D, et al. Serious morbidity associated with misuse of over-the-counter. codeine-ibuprofen analgesics: a series of 27 cases. Med J Aust 2010;193:294-6.

29. Dutch M. Nurofen Plus misuse: an emerging cause of perforated gastric ulcer. Med J Aust 2008;188:56-7.

30. Robinson RC, Gatchel RJ, Polatin P, et al. Screening for problematic prescription opioid use. Clin J Pain 2001:17:220-8.

31. Health NCCfM. Drug misuse: opioid detoxification. The NICE Guideline. London: Royal College of Psychiatrists and the British Psychological Society, 2008. 